\title{
Mansardas solares por optimización paramétrica y fabricación digital para viviendas NZE
}

\author{
Solar attic by parametric optimization and digital fabrication for NZE dwellings \\ - Lorena Troncoso Valencia \\ Universidad del Bío-bío, Chile \\ lorena618@gmail.com \\ - Alberto Nope Bernal \\ Universidad del Bío-bío, Chile \\ albertonope@gmail.com \\ - Rodrigo García Alvarado \\ Ricardo Arellano \\ Universidad del Bío-bío, Chile \\ Universidad del Bío-bío, Chile \\ rgarcia@ubiobio.cl \\ its.arellano@gmail.com
}

\begin{abstract}
In order to support the usual enlargement of houses in developing countries and proper integration of renewable sources, this paper exposes a parametric design of attic with insulated timber boards and integrated solar panels. The proposal is based on urban map of solar potential available on-line (www.msc.ubiobio.cl), that for single houses suggest a solar attic customized to each dwelling shape and orientation, with industrialized timber construction elements. The calculation of optimal volume by house is developed with a multi-objective genetic algorithm (NSGA-II) and dynamic simulation, which provides different buildings alternatives with digital manufacturing.
\end{abstract}

Keywords: Solar Energy, Timber Building, Housing, Genetic Algorithm, Building Integrated Solar Energy

\section{Introducción}

La mayoría de la población en Chile, y en muchos países en desarrollo, la población vive en viviendas aisladas que generan un alto consumo energético y poseen bajo desempeño ambiental. Especialmente en las latitudes medias con fuertes demandas de calefacción pero, a la vez reciben importantes niveles de radiación durante el año. Nuevas tecnologías de paneles fotovoltaicos, térmicos e híbridos pueden integrarse en cubiertas inclinadas y aportar de manera combinada energía para el consumo electricidad, calentar agua caliente y calefacción para los recintos. Logrando una auto-generación de sus demandas que permita alcanzar el concepto de vivienda de cero-energía (NZE). Las ampliaciones de las viviendas son una necesidad recurrente que pueden ser financiadas con créditos hipotecarios de menor tasa de interés. Sin embargo requieren techumbres adecuadamente orientadas y altos costos de instalación. Por lo que se plantea un sistema de cobertura adaptable a distintas viviendas, que otorgue una adecuada recolección solar y genere también espacios habitables. Para lo cual se desarrolla una programación paramétrica con optimización por algoritmo genético y una estrategia de fabricación digital que permita proponer techumbres variables y eficientes energéticamente, con bajos costos de ejecución. Estableciendo una novedosa integración de generación de la forma, análisis energético y construcción industrializada mediante sistemas digitales.

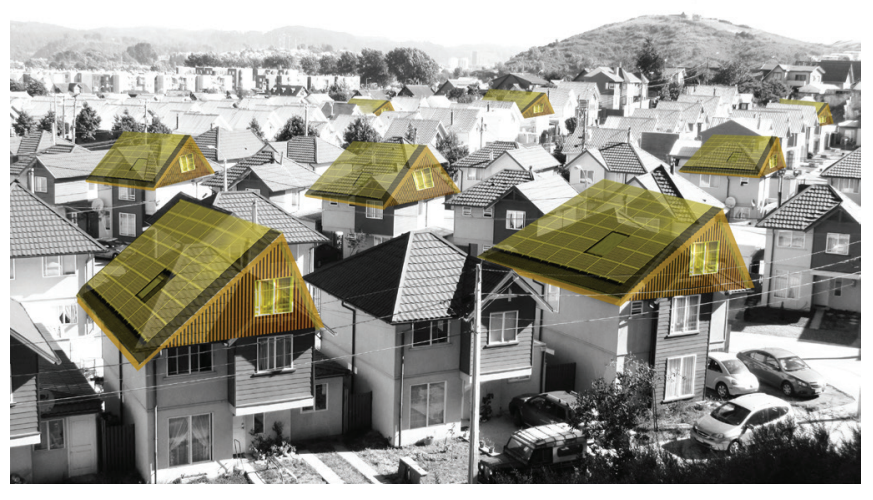

Figura 1: Imagen objetivo.

\section{Metodología}

Estudio previo potencial solar en Concepción Chile

Se efectuó un catastro de viviendas en el área urbana de la principal área metropolitana en el sur de Chile (comuna Concepción). Registrando sus demandas energéticas en calculadas según el número de personas y superficie de las edificaciones. Se generaron modelos volumétricos representativos según tipologías residenciales, para analizar la capacidad de captación solar en techos y fachadas. Se determinó la cantidad de radiación que recibe la ciudad, 
considerando porcentajes de sombra por topografía y edificaciones. Con esa información se propusieron sistemas de captación solar térmico, fotovoltaico e hibrido por tipologías residenciales. Esta estimación fue implementada en sistemas SIG. De esta manera los residentes pueden consultar sus potencialidades de instalación y beneficios energéticos esperados. Cabe señalar que se concluyó que en la mayoría de las viviendas unifamiliares poseen la capacidad suficiente en techumbre para autoabastecerse energéticamente, con una adecuada orientación. Entendiendo esto se realizó un catastro de las formas de las viviendas, para determinar rangos mínimos y máximos de perfiles de plantas sobre los cuales se montaran las mansardas. Por otro lado, también se instaló una plataforma en el que se estudian con distintas tecnologías solares, además de un piranómetro para medir la captación solar. En ella se está verificando el desempeño de paneles fotovoltaicos conectados a la red o independientes, térmicos con termosifón y bomba de calor, e híbridos con agua, en su recolección eléctrica y térmica, que es el primer sistema de este tipo instalado en el país.

\section{Programación paramétrica de las mansardas}

El diseño de la mansarda solar está basado en un algoritmo genético y simulación dinámica de modelos paramétricos. Desarrollando un análisis multi-objetivo, bajo una estructura NSGA-II (Non Dominated Sorted Genetic Algorithm), que permite ponderar de manera independiente distintas condiciones, aproximándose al pensamiento natural del diseñador o residente (Turrin et al, 2011; Mendez, 2013; Weng et al, 2014). La función objetivo del algoritmo de la mansarda solar está orientada a definir un volumen que cumpla tres objetivos integrados;

a. Maximizar la energía recibida a través de paneles solares integrados en su faldón de cubierta mejor orientado.

b. Maximizar la superficie habitable del interior para dar cabida a más recintos.

c. Minimizar la longitud de los elementos estructurales para disponer los menores costos de construcción.

Considerando como parámetros iniciales; la extensión del volumen constructivo de la vivienda en sus dos sentidos principales (ancho y profundidad), asumiendo salientes menores, o sea el perímetro de la casa sobre el cual se puede montar la mansarda. Como también su orientación respecto al sol. Las demandas energéticas se estiman de acuerdo a la superficie total de la vivienda (para lo que se solicita también la cantidad de pisos), y la provisión de energía capturada mediante tecnologías fotovoltaicas, térmicas o hibridas (combinadas), de acuerdo a la eficiencia de productos disponibles. La integración de paneles con una ampliación residencial pretende abordar ambas acciones con la posibilidad de financiamiento hipotecario con bajas tasas de interés y buena recuperación en el tiempo al reducir los gastos domésticos en servicios.

La programación está desarrollada en lenguaje Python, con JavaScript para la interfaz gráfica, la simulación dinámica se efectúa con EnergyPlus y la exportación de la geometría en DXF (Miller y Hersberger, 2013). Con una estructura de procesamiento por lotes en capas para la interfaz web, procesamiento (que desarrolla el análisis paramétrico y la consulta al motor energético), y una tercera capa de datos con una base y generación de archivos. De este modo, según los datos de entrada se desarrolla un modelo geométrico de la mansarda (un prisma triangular de base según las medidas principales), en que se analizan evolutivamente (seleccionando individuos en cada generación) el ángulo de inclinación lateral en el costado más orientado al norte.

Evaluando en cada caso la cantidad de superficie habitable generada por la forma (con altura mínima de 1,4 mts. y máx. de 2,4 mts. y restricciones laterales), como también la extensión de piezas moduladas en todas sus caras. A la vez que se envía el modelo a simulación para recabar su magnitud de radiación en la superficie y calcular generación según una tabla de eficiencia y paneles, en relación a la demanda estimada por superficie. Este análisis fue calibrado para un tiempo de procesamiento eficaz en 8 poblaciones y 6 generaciones, estableciendo soluciones dentro de un frente óptimo que ponderan las tres condiciones de optimización.

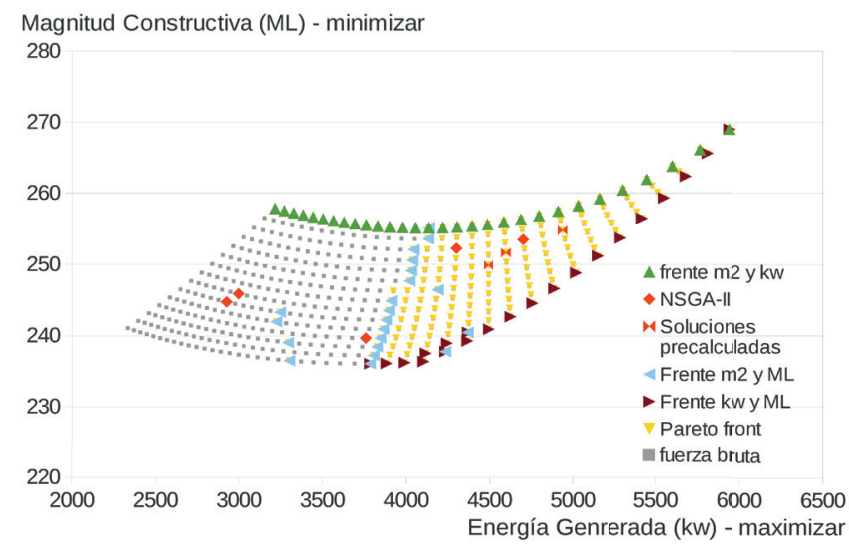

Figura 2: Grafico de resultados.

En una comparación con análisis por "fuerza bruta" (todos los casos posibles) se advierte que las soluciones NSGA-II se encuentran en áreas de optimización entre los distintos factores (Figura 2) aunque no constituyen las alternativas más óptimas en alguno en particular (el frente de optimización). Esto permite equilibrar factores y también ofrecer diferentes soluciones aproximadas (es decir no un diseño único), en las cuales el diseñador o residente, pueda elegir para compatibilizar con otras consideraciones del caso, por ejemplo su composición volumétrica, relación con casas vecinas, conformación de los espacios interiores, etc.(Figura 3) 


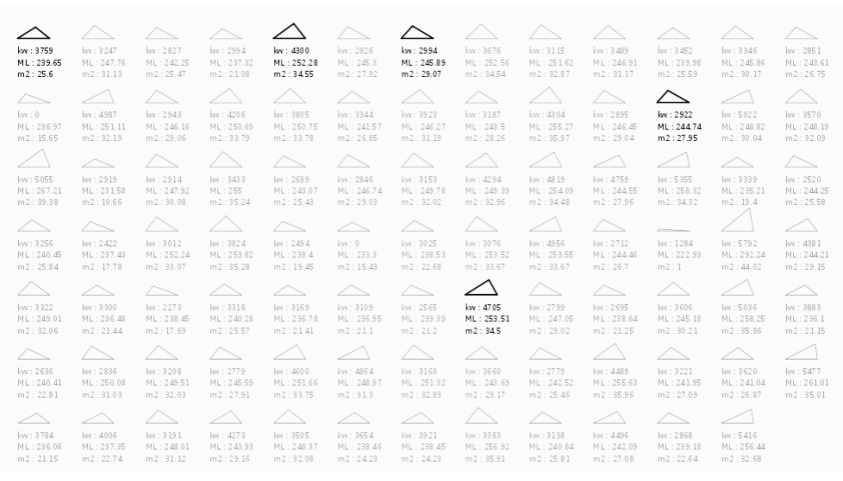

Figura 3: Análisis de perfiles.

Permitiendo posteriormente realizar la generación de un informe constructivo, con la planimetría básica de construcción y datos de los elementos requeridos.

\section{Aplicación en casos existentes.}

Como aplicación de la programación paramétrica, se sometieron a estudio 4 casos de viviendas existentes, todas con dos pisos y diferentes orientaciones solares. De un un mismo conjunto de tomaron 2 viviendas aisladas con diferente configuración, una vivienda pareada y una vivienda continua. Por cada una de ellas se definió el polígono base de la planta y se analizó por cada costado, revisando las dos posibles opciones constructivas resultantes de las mansardas.

$\mathrm{Si}$ bien los casos existentes tienen pequeños salientes, se definió como polígono regular la base a analizar ya que al ser más definido se evitan segundos faldones menores en la cubierta. Además se considera en los casos un alero de 60 $\mathrm{cm}$ en el sentido de la dirección de ángulo del techo, con esta estrategia se genera un aumento en el área útil interior y la superficie de captación. La Figura 4 en la primera columna muestra los casos analizados con distintas orientaciones; la segunda columna muestra bases esquemáticas según lado de ejecución y en la tercera columna se muestran los esquemas 3D de la mansarda propuesta provenientes de un DXF que genera la programación.

\section{Alternativas constructivas}

Actualmente las estructuras constructivas en Chile se clasifican en 8 tipos, estas varían desde la clase A, con estructura soportante en acero hasta la $\mathrm{H}$ con paneles ligeros. Se asumen capacidades estructurales básicas de la casa para resistir esta ampliación (muros interiores arriostrados cada $3 \mathrm{mts}$.), En momento de analizar los perfiles existentes también se revisaron los tipos de estructuras que aplican como base para las mansardas. Se determinó que hasta la clasificación E, con estructura soportante de madera, a partir de cierta escuadría es apta para soportar la mansarda. Se presume que algunos casos de viviendas existentes necesitarían refuerzos. Para estructurar las mansarda, se han realizado propuestas de cuatro sistemas constructivos; paneles, casetones, tijerales módulos estructurales. Las siguientes imágenes muestran el desarrollo de dos de ellos.

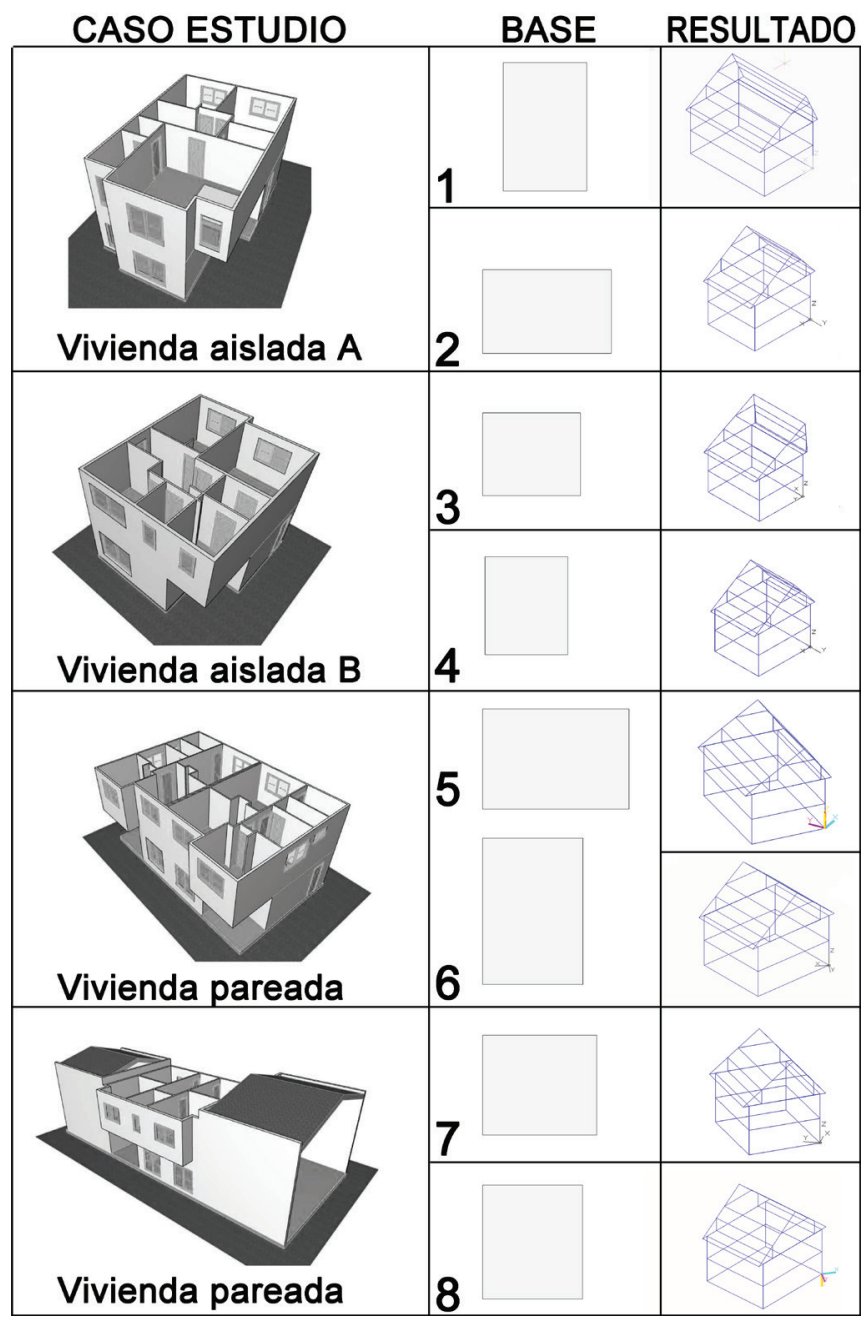

Figura 4: Casos analizados, bases rotadas y propuestas formales a partir de la programación paramétrica.

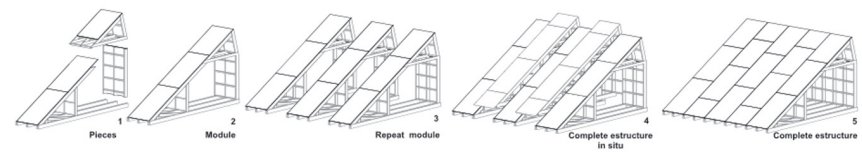

Figura 5: Alternativa con módulo de sección extruida.

Como se muestra en la Figura 5, se propone prefabricar la sección y a amplíe la sección conforme lo requiera la base existente. La segunda propuesta en Figura 6 es conformar la mansarda por paneles prefabricados y montados en terreno. Esta propuesta es más fácil de transportar y no requiere mayores maquinarias para montar el proyecto.

En ambos casos, los módulos vienen con la aislación térmica incorporada, in situ solo se instala el revestimiento de cubierta y lo la tecnología solar. La incorporación de los equipos para el funcionamiento de los sistemas híbridos, variara en cada caso, dependiendo de la disponibilidad de espacio en la vivienda base. 


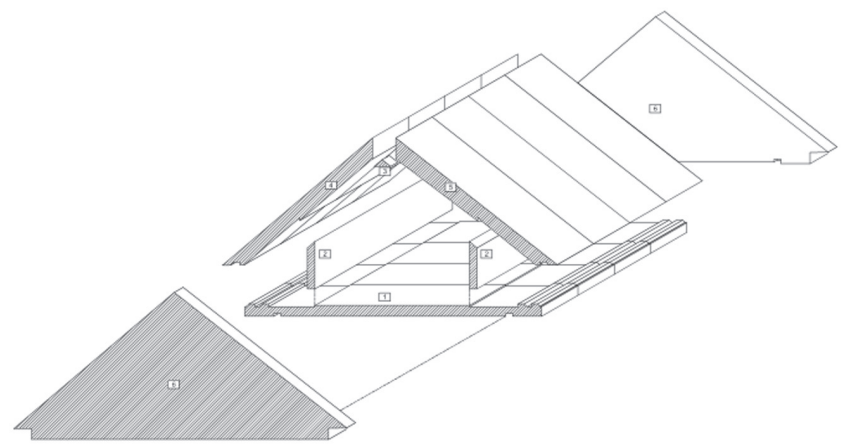

Figura 6: Alternativa con paneles aislantes de madera.

\section{Prototipo demostrativo a escala}

A modo de promover el uso y la tecnología solar en la población se están desarrollando modelos a escala y una sección de un prototipo a tamaño real como se muestra en la Figura 7 para probar los equipos y el proceso constructivo. Se considera una estructura soportante de tijerales con tableros de madera laminada, procedimiento constructivo que genera un menor impacto ambiental y una terminación interior más cálida por su apariencia natural. También mejoran las condiciones térmicas y acústicas que generan una menor demanda energética.

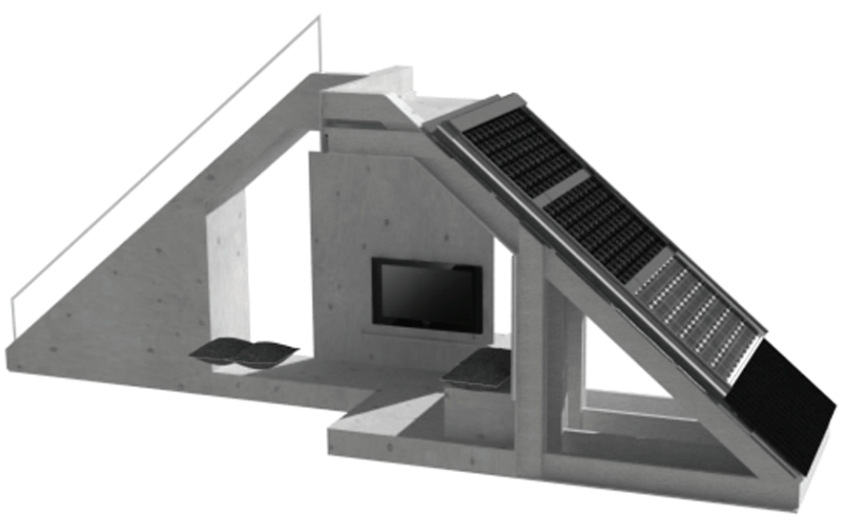

Figura 7: Prototipo a escala con tecnología solar.

\section{Futura implementación en línea}

Como una manera de incentivar a la población en el uso de energías renovable y considerando la iniciativa de la mansarda solar como un potencial proyecto de ampliación y generación energética para viviendas existentes, se está desarrollando una plataforma en línea en la cual cualquier usuario puede estudiar su mansarda con sus beneficios energéticos y espaciales según su propia vivienda (ver Figura 8).

Esta plataforma contiene el código de la programación paramétrica y mediante tres simples pasos que recogen la información de la vivienda existente referidos a las dimensiones de la vivienda donde se montara la mansarda, orientación respecto al sol y altura de pisos, el usuario obtiene un PDF en el cual se le entregan los resultados físicos energéticos y constructivos de la mansarda propuesta para la vivienda en cuestión (ver Figura 9).

Los resultados que se entregaran al usuario son el perfil físico, con el ángulo de inclinación, un 3D con la volumetría básica que incluye la morfología de la vivienda base. Estos datos gráficos se complementan con las producciones energéticas, áreas de paneles y posibles ahorros anuales.

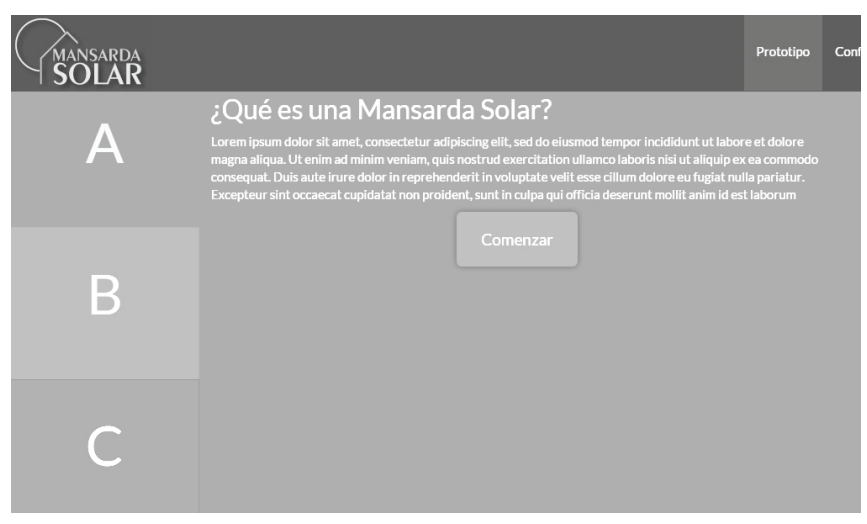

Figura 8: Sugerencia de web para difusión pública.
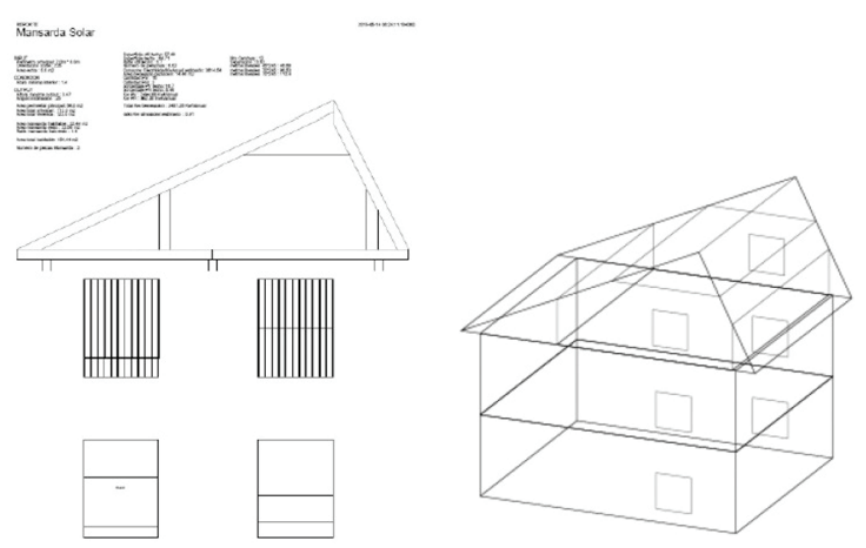

Figura 9: Imagen gráfica de los resultados en PDF.

\section{Resultados}

La optimización paramétrica desarrollada ha permitido proponer diseños de mansarda con paneles híbridos integrados que otorgan un incremento del $25 \%$ del área habitable de la vivienda con dos a cuatro recintos adicionales. Además de provisión de energía eléctrica que supera en cinco veces la demanda regular, y sobre la mitad de los requerimientos de ACS y calefacción (un 30\% en invierno y $80 \%$ en primavera/verano), que puede ser complementado con la retribución eléctrica a la red en los periodos más favorables, es decir alcanzado un desempeño cercano al cero-energía por vivienda (NZE). Mediante el desarrollo de construcción industrializada y el catastro efectuado se puede estimar que la solución es aplicable en cerca de la mitad de las viviendas de la zona, con un alcance relevante en la población de latitudes medias, las cuales combinan altas demandas energéticas con una relevante radiación solar. 


\section{Discusión}

La programación debe ser refinada en su interfaz gráfica para implementar de manera gratuita en un sitio web de difusión y verificar sus resultados de acuerdo a la aplicación y desempeño de sistemas instalados al menos un año. La aplicación del diseño propuesto depende de la participación de empresas constructoras, instaladores y proveedores adecuadamente capacitados y que confirmen la implementación dentro de los costos estimados. Considerando también la aceptación de los créditos hipotecarios posibles para masificar la propuesta dentro de condiciones financieras accesibles para la mayoría de la población. Pero se ha logrado establecer un procedimiento informático original para apoyar un crecimiento habitacional sustentable con un diseño masivo variable (mass-customization). Por otro lado, la propuesta promueve una ampliación superior de las viviendas que otorga un nuevo perfil y perspectiva residencial, con interiores más cálidos y mayores visiones del entorno, además de integrar arquitectónicamente la auto-generación energética que otorga independencia y resiliencia urbana. Diseminando una conciencia ambiental en la población y una reducción efectiva de los efectos del alto consumo energético, mediante nuevas herramientas profesionales que integran capacidades de diseño, análisis y fabricación digital.

\section{Agradecimientos}

Los autores agradecen a AKA-ERNC 007, CD-INes Prototipos, EnerSolar, Solimpek, Paulina Wegertseder, Manuel Suazo, Carola Vidal, Hannu Hirsi, Hannu, Pekka Heikkinen por su colaboración con el proyecto.

\section{Referencias}

Athienitis, A. K., Bambara, J., ONeill, B., \& Faille, J. 2011. Design and performance of a photovoltaic/thermal system integrated with transpired collector. Solar Energy, 117(1), 403-410.

Celis, F., Garcia, R., Trebilcock, M. T., Escorcia, O., \& Diaz, M. 2012. Análisis energético de las viviendas del centro-sur de Chile. Arquiteturarevista, 8, 62-75
Chow, T. 2010. A review on photovoltaic / thermal hybrid solar technology. Applied Energy, 87(2), 365-379

De la Barrera, C. 2010. Algoritmos Genéticos como Estrategia de Diseño en Arquitectura, Ph.d. Thesis, Barcelona: Universidad Politécnica de Catalunya, $206 \mathrm{p}$.

García, R.; Donath, D.; Gonzalez, L. 2009. Growth patterns in incremental self-build housing in Chile. Open House International Vol. 4, n³4. 2009, pp 18-25.

García, R. 2012. Parametric Development of Variable Roof Structures with Central Supports (Tulips), Nexus 2012 Relationships Between Architecture and Mathematics, Politécnico do Milano, Milán.

Hachem, C. 2012. Investigation of Design Parameters for Increased Solar Potential of Dwellings and Neighborhoods. Concordia University

Kieran s. y Timberlake J., Refabricating Architecture, How Manufacturing Methodologies Are Poised to Transform Building Construction, Ed. McGraw-Hill, New York, 2004.

Lund, P. 2012. Large-scale urban renewable electricity schemes. Energy Conversion and Management, 63.

Mendez, Tomás. , Computational Search in Architectural Design, 2013 , Politecnico di Torino, Italia.

Meredith et al; 2008, From Control to Design; Parametric/Algorithm Architecture", Barcelona: Actar.

Miller, Clayton; Hersberger, Christian. Automation of common building energy simulation workflowsusing Python, 2013, Conference: 13th Conference of the International Building Performance Simulation Association.

Poblete, M.C., 1991. Sistemas estructurales de madera, Concepción: Universidad del Bio-Bio.

Turrin, M.; R. Stouffs and S. Sariyildiz, 2010. Parametric Design of the Vela Roof, en 5th International Conference of the Arab Society for Computer Aided Architectural, Fez (Morocco), pp. 231-240.

Turrin,M.,von Buelow, P., Stouffs,R.,(2011), Design explorations of performance driven geometry in architectural design using parametric modelling and genetic algorithms. Advanced Engineering Informatics, 24(5), Elsevier, pp 656-675.

Weng, Zhenzhou et al. ,The practical optimisation of complex architectural forms. 2014, University of Bath, UK. 